\title{
External magnetic field promotes homing of magnetized stem cells following subcutaneous injection
}

\author{
Yu Meng ${ }^{1 \dagger}$, Changzhen Shi ${ }^{2 \dagger}$, Bo Hu ${ }^{1}$, Jian Gong ${ }^{3}$, Xing Zhong ${ }^{3}$, Xueyin Lin ${ }^{3}$, Xinju Zhang ${ }^{4}$, Jun Liu ${ }^{4}$, \\ Cong $\mathrm{Liu}^{4}$ and $\mathrm{HaO} \mathrm{Xu}^{3^{*}}$
}

\begin{abstract}
Background: Mesenchymal stem cells (MSCs) are multipotent stromal cells that have the ability to self-renew and migrate to sites of pathology. In vivo tracking of MSCs provides insights into both, the underlying mechanisms of MSC transformation and their potential as gene delivery vehicles. The aim of our study was to assess the ability of superparamagnetic iron oxide nanoparticles (SPIONs)-labeled Wharton's Jelly of the human umbilical cord-derived MSCs (WJ-MSCs) to carry the green fluorescent protein (GFP) gene to cutaneous injury sites in a murine model.
\end{abstract}

Methods: WJ-MSCs were isolated from a fresh umbilical cord and were genetically transformed to carry the GFP gene using lentiviral vectors with magnetically labeled SPIONs. The SPIONs/GFP-positive WJ-MSCs expressed multipotent cell markers and demonstrated the potential for osteogenic and adipogenic differentiation. Fifteen skininjured mice were divided into three groups. Group I was treated with WJ-MSCs, group II with SPIONs/GFP-positive WJ-MSCs, and group III with SPIONs/GFP-positive WJ-MSCs exposed to an external magnetic field (EMF). Magnetic resonance imaging and optical molecular imaging were performed, and images were acquired 1, 2, and 7 days after cell injection.

Results: The results showed that GFP could be intensively detected around the wound in vivo $24 \mathrm{~h}$ after the cells were injected. Furthermore, we observed an accumulation of WJ-MSCs at the wound site, and EMF exposure increased the speed of cell transport. In conclusion, our study demonstrated that SPIONs/GFP function as cellular probes for monitoring in vivo migration and homing of WJ-MSCs. Moreover, exposure to an EMF can increase the transportation efficiency of SPIONs-labeled WJ-MSCs in vivo.

Conclusions: Our findings could lead to the development of a gene carrier system for the treatment of diseases.

Keywords: Mesenchymal stem cells (MSCs), Stem cells, UC, Magnetic resonance imaging (MRI), Rat

\section{Background}

There is significant potential for the use of mesenchymal stem cells (MSCs) in cell therapy [1]. However, their clinical application still faces various challenges, such as the fact that an efficient strategy for stem cell homing to target sites has not yet been identified. Several other factors limit the clinical application of stem cells, including the time and method of drug administration, cell

\footnotetext{
* Correspondence: xuhaodoc@163.com

${ }^{\dagger}$ Equal contributors

${ }^{3}$ Department of Nuclear Medicine, the First Hospital Affiliated to Jinan University, No. 613 Huangpu West Road, Guangzhou 510630, China Full list of author information is available at the end of the article
}

concentration, the transmission medium, and cell homing $[1,2]$. Homing of stem cells is achieved through direct local injection, local perfusion, and systematic administration. However, intravenous injection of stem cells results in the accumulation of a significant number of cells in the lungs and spleen, with a very low percentage of cells reaching the arterial system (about 5\%), and an even lower percentage reaching the target organ $(0.0005 \%)[2,3]$. Simple direct injections and local organ perfusions are limited to superficial organs or to organs directly connected to the main artery. In fact, although an intra-arterial injection ensures highest cell numbers for transplant, this method of stem cell transplantation 
increases the death rate by $41 \%$, with animals succumbing to arterial embolism [4]. Therefore, an important prerequisite for treatments is to transplant MSCs with a differentiation potential directly to the target area.

Compared with traditional preparations, magnetic targeted drug delivery systems, which have been studied for years [5-8], are characterized as methods for improving drug targeting, enhancing the curative effect of drugs, and decreasing toxic side effects. Superparamagnetic iron oxide nanoparticles (SPIONs) are an excellent transmission medium based on the magnetic targeted drug delivery system [9-14]. Recent studies have revealed that labeling stem cells with magnetic nanoparticles for magnetic resonance imaging (MRI)-mediated tracking of stem cells has evolved. An improved curative effect on common carotid artery injuries was observed using magnetized endothelial progenitor cells, obtained from in situ intraarterial treatment of spinal cord injured animals, using a magnetic field to direct the stem cells [15]. Recently, studies have used anionic magnetic nanoparticles to load endothelial progenitor cells, and have successfully controlled cell movement in the vessel network using a magnetic field [16]. Although the results are exciting, most of these studies involved the use of a constant electromagnetic field or an internal magnetic field.

Studies involving noninvasive external magnetic fields (EMFs) with the use of permanent magnets are rare. The superparamagnetism of SPIONs can be utilized to bring about directional movement of magnetized stem cells under the influence of an EMF. In the present study, human umbilical cord MSCs were transfected with SPIONs and green fluorescent protein (GFP), and injected into the subcutaneous tissues of nude mice, specifically into partial cells at some cell intervals, following a skin injury. In summary, although some studies have shown that noninvasive EMFs can increase magnetized cell homing following an intra-arterial injection, intravenous injection, or intrathecal injection, no evidence has been provided showing the same effect following a subcutaneous injection. The current study tested the hypothesis that an EMF can promote homing and guide the magnetized stem cells to make rapid directional movements, following subcutaneous injection. MRI as well as fluorescence imaging was used to track the stem cells in vivo. Our findings demonstrated an improved method of injury treatment, using MSCs as drug or gene carriers, which can also be applied for directional drug treatment or gene therapy, both of which have a significant importance in the clinical setting.

\section{Methods}

\section{Cell culture}

The study was approved by the regional medical ethical review board (Jinan University and Shenzhen people's hospital). After obtaining a written informed consent, the human umbilical cord Wharton's Jelly-derived MSCs (WJ-MSCs) were isolated as described previously [15], from the umbilical cords of full-term newborns, delivered at the Shenzhen People's Hospital, Guangdong, China. Cells were cultured in low glucose-DMEM (Hyclone, Logan, Utah, USA) containing $2 \mathrm{ng} / \mathrm{mL}$ of basic fibroblast growth factor (bFGF) and 10\% fetal bovine serum (FBS) (Gibco, Gran Island, NY, USA), and maintained at $37{ }^{\circ} \mathrm{C}$ in a humidified atmosphere of $5 \%$ $\mathrm{CO}_{2}$. The medium was replaced every 3 days, and the human umbilical cord-derived MSCs (HUCMSCs) were collected by trypsin $(0.25 \%$, Invitrogen, USA) digestion. All experiments were performed using MSCs at 3-5 passages [17].

\section{Flow cytometry analysis}

Passage $3 \mathrm{WJ}$-MSCs were trypsinized, dissociated into a single cell suspension, and allowed to reach $60 \%$ confluency. Cells were then rinsed with phosphate buffered saline (PBS) and incubated with anti-human CD73-PE (BioLegend, 344,004), anti-human CD105-PE (BioLegend, 323,206), anti-human CD90-PE (BioLegend, 328,110), anti-human CD34-PE (BioLegend, 343,506), and anti-human CD45-FITC (BioLegend, 304,006) for $15 \mathrm{~min}$ at room temperature. After incubation, the cells were rinsed with PBS, read on a FACSCalibur (BD, USA) flow cytometer, and analyzed using the WinMDI 2.8 software. Mouse IgG1-PE (BioLegend, 400,114) and mouse IgG1-FITC (BioLegend, 400,107) were used as the isotype controls.

\section{Osteogenic differentiation}

At approximately $80 \%$ confluency, WJ-MSCs were rinsed with PBS and cultured in osteogenic differentiation medium (Gibco, Gran Island, NY, USA). The medium was changed twice a week, and after 3 weeks, the cells were washed with PBS and fixed in $4 \%$ paraformaldehyde for $30 \mathrm{~min}$. The cells were then stained with $0.1 \%$ Alizarin Red S water solution for $30 \mathrm{~min}$.

\section{Adipogenic differentiation}

At $80 \%$ confluency, WJ-MSCs were rinsed with PBS and cultured in adipogenic differentiation medium (Gibco, Gran Island, NY, USA). The medium was changed twice a week, and after 3 weeks, the cells were washed with PBS and fixed in $4 \%$ paraformaldehyde for $30 \mathrm{~min}$. The cells were then stained with $0.3 \%$ Oil Red O solution for $30 \mathrm{~min}$.

\section{Lentiviral transfection of WJ-MSCs}

A lentiviral vector (Fitgene, Guangzhou, China) expressing GFP with a cis-acting element, CMV-GFP-puro, was packaged and used to infect the WJ-MSCs, according to 
the manufacturer's protocol. The transduced cells were grown in low glucose-DMEM containing $2 \mathrm{ng} / \mathrm{mL} \mathrm{bFGF}$, $10 \%$ FBS, and $400 \mu \mathrm{g} / \mathrm{mL}$ puromycin. Puromycinresistant WJ-MSCs, overexpressing GFP, were obtained after 3 days of puromycin selection. Stable clones were identified by the expression of GFP protein [16]. The efficiency of the target cells was estimated by fluorescence microscopy using an inverted fluorescence microscopethe GFP positive cells in each field (10X) visible/all the cells in each field (10X).

\section{Labeling of WJ-MSCs with SPIONs}

Approximately $1 \times 10^{3}$ genetically modified WJ-MSCs were seeded into each well of a 24-well plate. After $12 \mathrm{~h}$ of incubation in MSC growth medium, the cells were magically labeled with SPIONs $(25 \mu \mathrm{g} / \mathrm{mL}$; SPIONsMSCs) (Molday ION Rhodamine $\mathrm{B}^{\mathrm{rm}}$, BioPhysics Assay Laboratory, Inc., Worcester, MA, USA; CL-50Q02-6A$50)$ and complexed to poly-L-Lysine $(0.75-1 \mu \mathrm{g} / \mathrm{mL})$ (BioPhysics Assay Laboratory, Inc., CL-00-01). The cells were then collected, washed twice in PBS, counted, and resuspended at the appropriate cell density for in vivo analyses.

\section{Cell vitality test}

For Trypan blue staining, $200 \mu \mathrm{L}$ of cells was aseptically transferred to a $1.5 \mathrm{~mL}$ clear Eppendorf tube, and incubated for $3 \mathrm{~min}$ at room temperature with an equal volume of $0.4 \%(w / v)$ Trypan blue solution prepared in $0.81 \% \mathrm{NaCl}$ and $0.06 \%(w / v)$ dibasic potassium phosphate. Cells were counted using a dual-chamber hemocytometer and a light microscope. Viable and nonviable cells were recorded separately, and the means of the two cell counts were pooled for analysis.

\section{Full-thickness skin defect model and cell transplantation} In total, 30 specific pathogen-free BALB/C nude mice were randomly divided into 3 groups: 10 mice in the control group, 10 mice in the group receiving SPIONsMSCs, and 10 mice in the group receiving SPIONsMSCs exposed to an EMF (SPIONs-MSCs + magnetic field). The full-thickness skin defect model was implemented in all experimental mice, and cell transplantation was performed at a $1.5 \mathrm{~cm}$ distance from the wound using a $1 \mathrm{~mL}$ syringe. The control group was injected with $200 \mu \mathrm{L} 0.9 \%$ normal saline, the SPIONs-MSCs group was injected with $2 \times 10^{6}$ SPIONs and GFP double-labeled MSCs, and the SPIONs-MSCs + magnetic field group was injected with $2 \times 10^{6}$ SPIONs and GFP double-labeled MSCs exposed to an EMF generated by a permanent magnet for $6 \mathrm{~h} /$ day. The effects of different EMF exposure times on stem cells were analyzed by the MTT assay and western blotting, by determining the apoptosis markers (Additional file 1: Figure S1). The full-thickness skin defect model was generated as follows: mice were anesthetized by an intraperitoneal injection of $10 \%$ chloral hydrate $(0.3 \mathrm{~mL} / 100 \mathrm{~g})$; next, following disinfection, a 4-mm diameter circular skin defect was created to the depth of the deep fascia on the back of the mice, near the double hind limb. After completion of stem cell transplantation, a representative animal from each group was immediately subjected to MRI and in vivo fluorescence examination for baseline analysis. Three mice per group were subjected to MRI and fluorescence imaging at $24 \mathrm{~h}, 48 \mathrm{~h}$, and 7 days post-cell transplantation, and the healed skin lesions were removed for pathological examination on day 7 . Healed wounds were identified by the presence of the following: wound closure, epithelium, proper activity intensity, no wound dehiscence, no ulceration, an appropriate time lapse after wound generation, the ability to tolerate a certain tension and pressure at the wound site, gradual fading of skin color at the wound site, and similarity of the wound skin color to that of the surrounding healthy skin to maintain the skin barrier integrity. All experimental procedures were approved by the Animal Experimentation Ethics Committee of First Hospital Affiliated to Jinan University, Guangzhou, China.

\section{In vivo injection of magnetic WJ-MSCs}

Mice were anesthetized with isoflurane (4\% induction, $1.5 \%$ maintenance). The wounds were exposed to a magnetic field of $0.5 \mathrm{~T}$ using a small permanent neodymium $($ FeNdB) magnet $(8 \times 2 \mathrm{~mm})$ for $6 \mathrm{~h} /$ day (Additional file 2: Figure S2). Subsequently, $2 \times 10^{6} \mathrm{WJ}-\mathrm{MSCs}$, which were previously magnetized using $25 \mu \mathrm{g} / \mathrm{mL}$ of SPIONs, were hypodermically injected (hypodermis) with $150 \mu \mathrm{L}$ of PBS. Control animals received an identical cell infusion without the magnet implantation. Magnets were placed for $6 \mathrm{~h}$ daily.

\section{In vivo MRI}

The animals used were BALB/C (nu/nu) nude mice $(n=3)$. One day, two days, or seven days after transplantation, the mice were anesthetized using chloral hydrate and underwent in vivo MRI (gradient echo scan), using a 3 T MRI scanner (Discovery MR750, GE Healthcare, USA). For image acquisition, a quadrature birdcage volume coil of $7 \mathrm{~cm}$ inner diameter was used. Axial images were taken with the following parameters: field of view $=4 \times 4 \mathrm{~cm}^{2}$, matrix size $=256 \times 256$, slice thickness $=1 \mathrm{~mm}, \mathrm{TE}=6 \mathrm{~ms}$, and $\mathrm{TR}=700 \mathrm{~ms}$. Following completion of the scan, the raw data and images were processed using the built-in professional software Discovery MR750, with each injected area defined as a unit, and using each of the 4 selected regions of interest (ROI) for measurements. MRI parameters included 
injection of cell volume, displacement, carrier-to-noise ratio (CNR), and signal-to-noise ratio (SNR).

\section{Fluorescence stereomicroscopy}

To assess the distribution of SPIONS-MSCs in vivo, anesthetized rats were imaged for GFP fluorescence using a whole-body imaging system (IVIS Lumina II, Caliper, France). Filters of $480 \mathrm{~nm}( \pm 10 \mathrm{~nm})$ and $505 \mathrm{~nm}( \pm 5 \mathrm{~nm})$ represented the excitation and emission signals, respectively. High-resolution images were captured directly on a computer and analyzed using Living Image software (Xenogen Corporation, Almeda, California, USA). Results were expressed as number of photons/s/ROI.

\section{Prussian blue staining and tissue specimens}

Prussian blue staining was performed to identify the SPIONs-MSCs. Cells were incubated for 30 min with $2 \%$ potassium ferrocyanide in $6 \%$ hydrochloric acid, and then counterstained with nuclear fast red for $30 \mathrm{~s}$. A blue color indicated the presence of iron within the cells, thereby corresponding to the SPIONs-MSCs. Similarly, in skin tissue sections, SPIONs appeared as blue precipitates in the cytoplasm and pink in the nucleus. Tissue specimens were frozen in optimal cutting temperature (OCT) compound (Sakura Finetek Inc., Torrance, California, USA) in liquid nitrogen, and $10-\mu \mathrm{m}$ sections were prepared using a cryostat microtome (CM1850; Leica Microsystems $\mathrm{GmbH}$ ).

\section{Statistical analysis}

The statistical significance of intergroup differences was assessed using the Student's $t$-test or ANOVA followed by Tukey's post hoc test. A $P$ value $<0.05$ was considered statistically significant at the $95 \%$ confidence level. All values in the bar and line graphs are expressed as mean \pm standard deviation (SD). The number of independent experiments analyzed has been stated in each figure legend.

\section{Results}

\section{Lentivirus infection and SPIONs labeling}

In our study, GFP was used for the in vivo tracking of WJMSCs. GFP was incorporated into a lentiviral vector containing independent puromycin expression frames. WJMSCs were isolated from fresh umbilical cords and cultured in MSC medium for several passages. Lentivirusinfected WJ-MSCs were selected in MSC medium with puromycin for 3 days. Stable clones were GFP positive (>99\%), as detected by fluorescence microscopy (Fig. 1a and b). GFP expression was observed under a fluorescence microscope. Using an inverted fluorescence microscope, we observed the HUCMSCs for a green fluorescence sig$\mathrm{nal}$ at $12 \mathrm{~h}$ post-transfection; however, the signal was weak and expressed only by a few cells. The number of GFPpositive cells increased constantly $24 \mathrm{~h}$ post-transfection, with 4-10 GFP-positive cells in each visual field (10X) at $48 \mathrm{~h}$, and more than $10 \mathrm{GFP}$-positive cells in each visual field (10X) at $72 \mathrm{~h}$. The GFP transfection efficiency with lentivirus infection was over 99\%. The GFP-positive WJ-

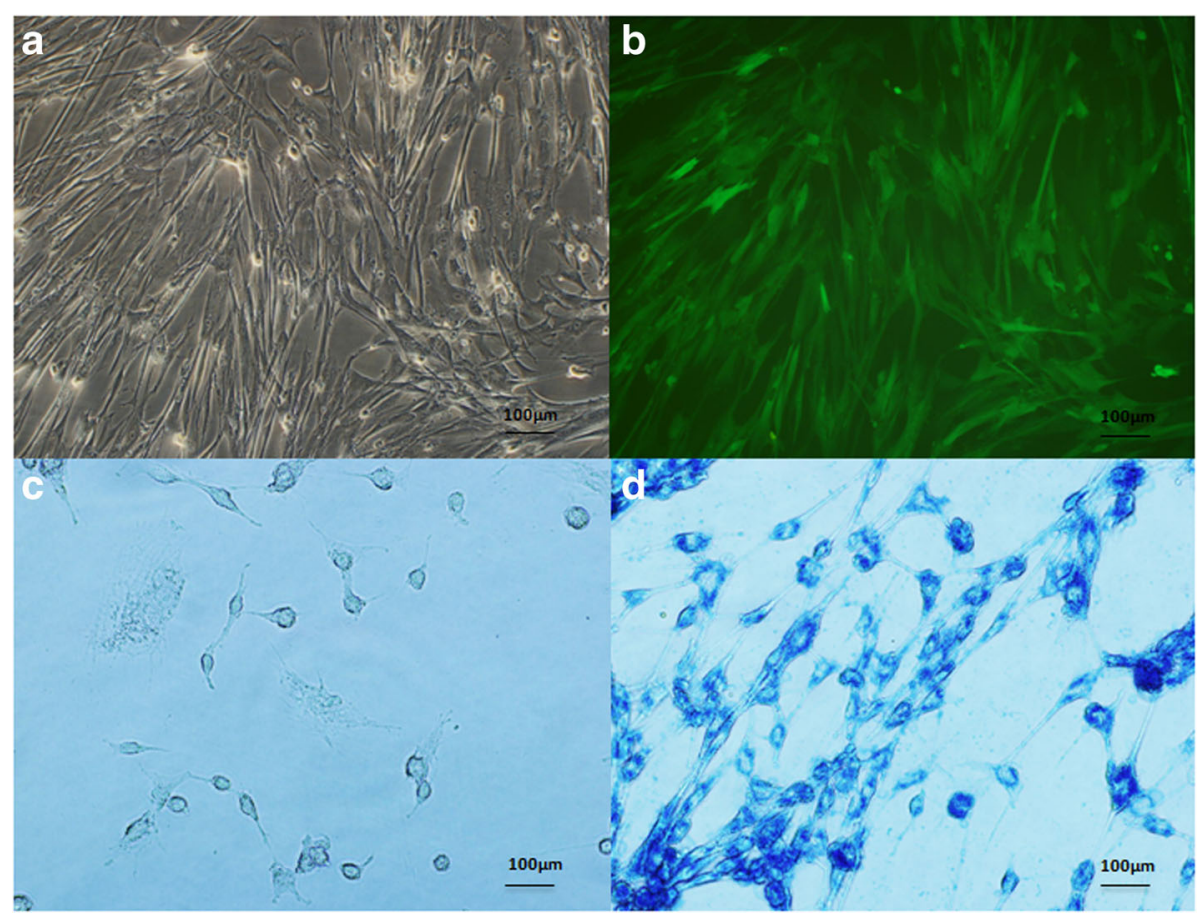

Fig. 1 WJ-MSCs labeled with GFP/SPIONs. GFP-positive cells under fluorescence microscope (a) and (b). Cells labeled with SPIONs (c) and (d) 
MSCs were then transfected with SPIONs, and the transfection efficiency was evaluated by Prussian blue staining. Results demonstrated that more than $80 \%$ of the cells were labeled with SPIONs (Fig. 1c and d).

\section{Immunophenotype, differentiation potential, and vitality of WJ-MSCs}

The immunophenotype of passage 3 WJ-MSCs, which represent typical fibroblastic cells, and GFP/SPIONspositive WJ-MSCs was evaluated by flow cytometry. The results showed that all cells expressed CD73, CD105, and CD90 (>95\%), but did not express CD34 or CD45 $(<2 \%)$ (Fig. 2). Furthermore, both untransfected WJMSCs and GFP/SPIONs-positive WJ-MSCs were evaluated for their osteogenic and adipogenic differentiation potential. After a 3-week induction under osteogenic conditions, these cells were stained with $0.1 \%$ Alizarin Red S water solution. Results showed that majority of the WJ-MSCs were alkaline phosphatase-positive, indicating their osteogenic differentiation potential (Fig. 3a

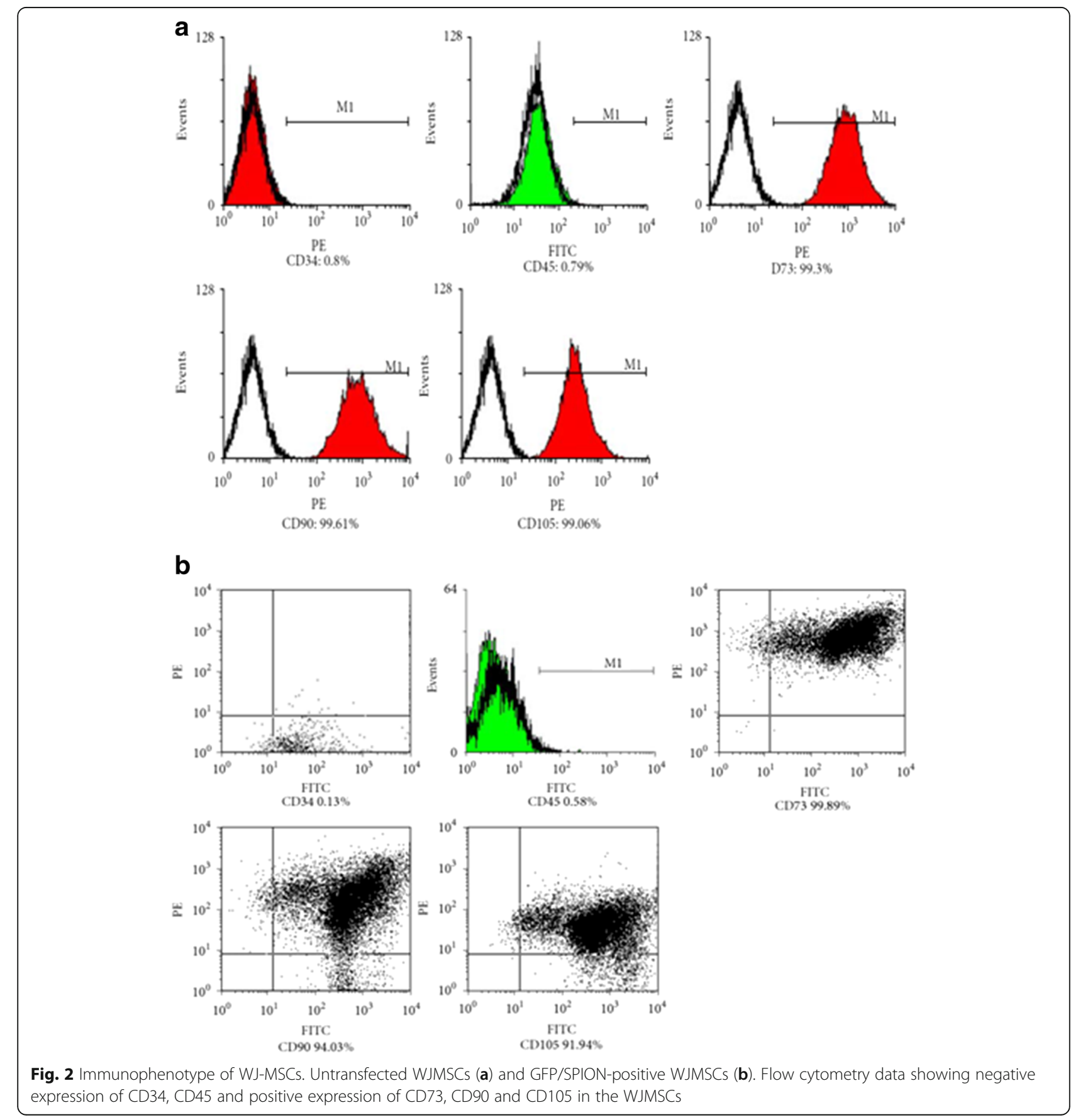




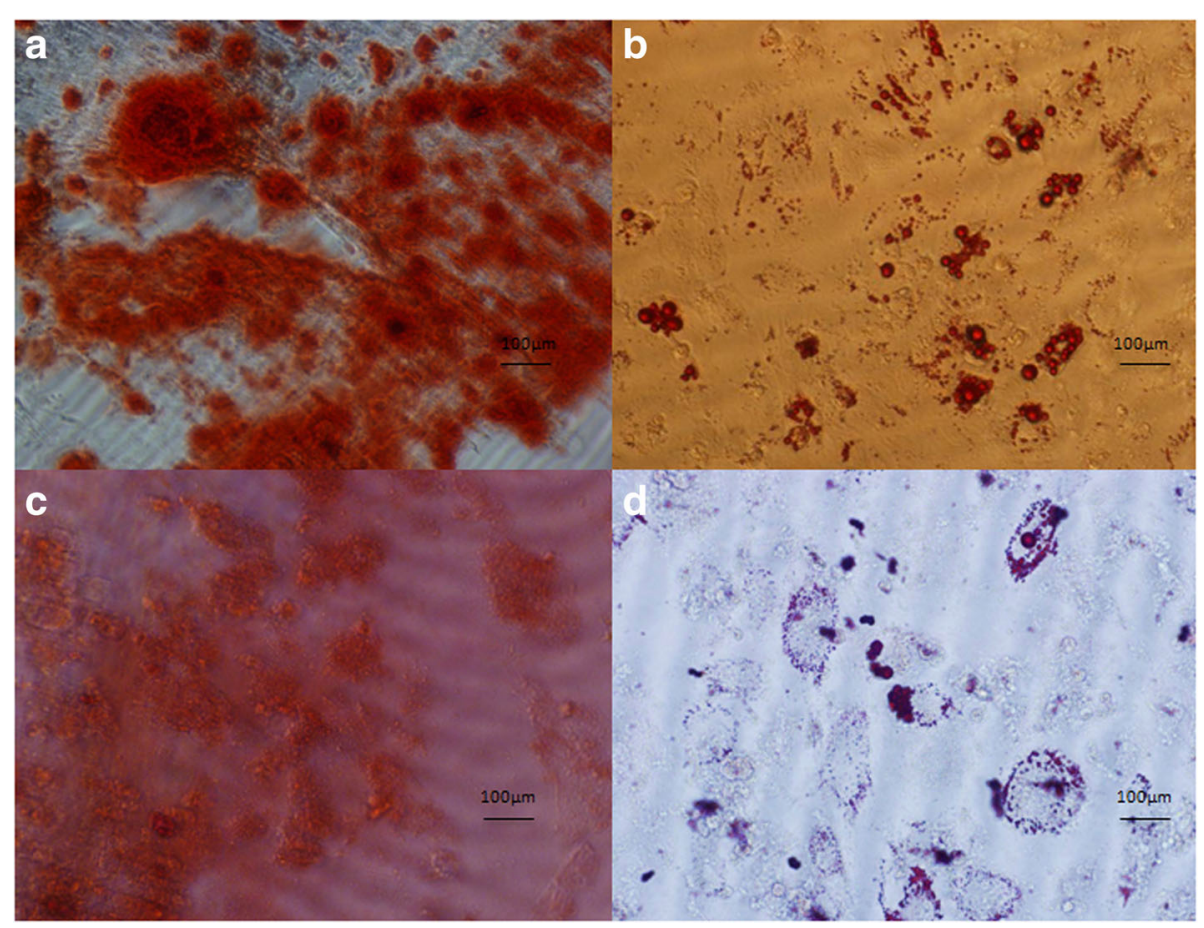

Fig. 3 Differentiation of WJMSCs. Osteogenic differentiation analysis of untransfected WJMSCs (a) and GFP/SPION-positive WJMSCs (c). Adipogenic differentiation analysis of untransfected WJMSCs (b) and GFP/SPION-positive WJMSCs (d)

and c). To assess their adipogenic differentiation potential, another culture plate of passage $3 \mathrm{WJ}-\mathrm{MSC}$ was incubated with adipogenic differentiation medium for 3 weeks and then stained with $0.3 \%$ Oil Red O. We observed that majority of the cells contained numerous Oil Red O-positive lipid droplets, indicating that WJ-MSCs underwent adipogenic differentiation. (Fig. $3 \mathrm{~b}$ and d). Growth of GFP/SPIONs-positive WJ-MSCs was seen in the two multiplication cycles; the first multiplication cycle started on day 3 and 4, and the second one in the first 4-7 days. Compared with the control group (HUCMSCs), there was no significant difference at all time points $(t=2.05, p>0.05)$ (Additional file 3: Figure S3).

\section{In vivo cell tracking using MRI}

GFP/SPIONs-positive WJ-MSCs were injected around the cutaneous wound areas in injured mice. MRI was performed at 0,24 , and $48 \mathrm{~h}$ and 7 days posttransplantation. As expected, GFP/SPIONs-positive WJMSCs were successfully directed to the subcutaneous areas of the skin under the influence of the magnetic gradient created by the implanted magnet (Fig. 4). The effect of the EMF on stem cell targeting was significant compared to WJ-MSCs without exposition to magnetic field. The 24-h MR images confirmed that more than $80 \%$ of SPIONs/GFP-labeled WJ-MSCs (low signal distribution) reached the trauma center within the first $24 \mathrm{~h}$. However, only low signals were detected around the wounds that were not exposed to the EMF. On day 7 , post-cell transplantation, MRI results demonstrated a hypointense signal distribution in the wound center of mice regardless of the magnetic field interference. However, the MRI parameters were not significantly different between the SPIONS-MSCs and SPIONs-MSCs + magnetic field groups. Parameters such as area, SNR, CNR, and displacement can be used as indicators of the concentration and movement speed of cell clusters. In the SPIONs-MSCs + magnetic field group, we observed a significantly reduced area (Fig. 5a) and an increased SNR, CNR, and displacement $24 \mathrm{~h}, 48 \mathrm{~h}$, and 7 days post-cell transplantation, particularly in the first $24 \mathrm{~h}$ (Fig. 5b). The CNR was significantly different between the two groups, especially at the $24 \mathrm{~h}$ time point (Fig. 5c). The highest value of SNR was observed at the 7 day time point in the two groups (Fig. 5d).

Moreover, we observed that the wound recovery rate was enhanced in the SPIONs/GFP-MSCs + magnetic field group as compared with the SPIONs/GFP-MSCs group and the control group. Qualitative skin analysis via Prussian blue staining and fluorescence imaging was performed to validate that the hypointense signals detected by MRI indeed corresponded to the presence of MSCs at the target site. The presence of blue cells (Prussian blue) was confirmed in matching skin injury areas, indicating the presence of MSCs in the skin tissue (Fig. 6). Both groups of SPIONs-MSCs, with or without 


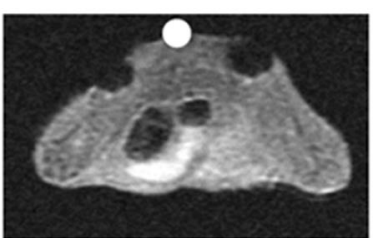

a

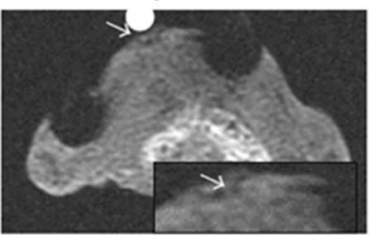

b

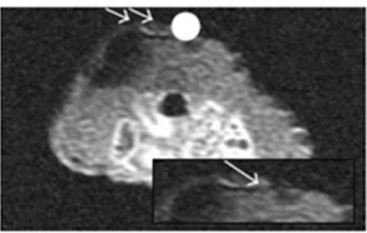

C

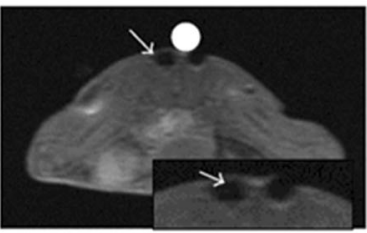

d

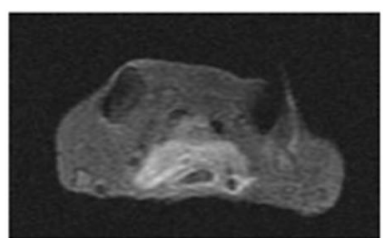

e

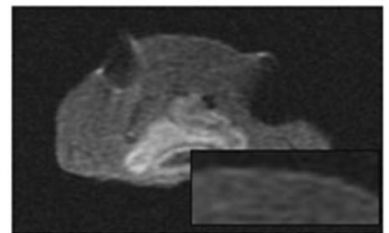

f

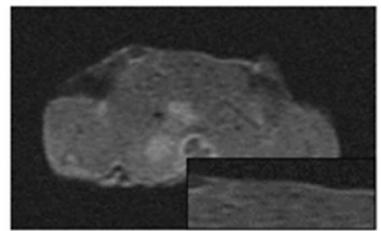

g

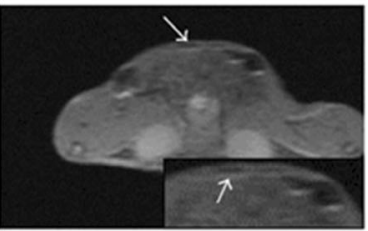

h
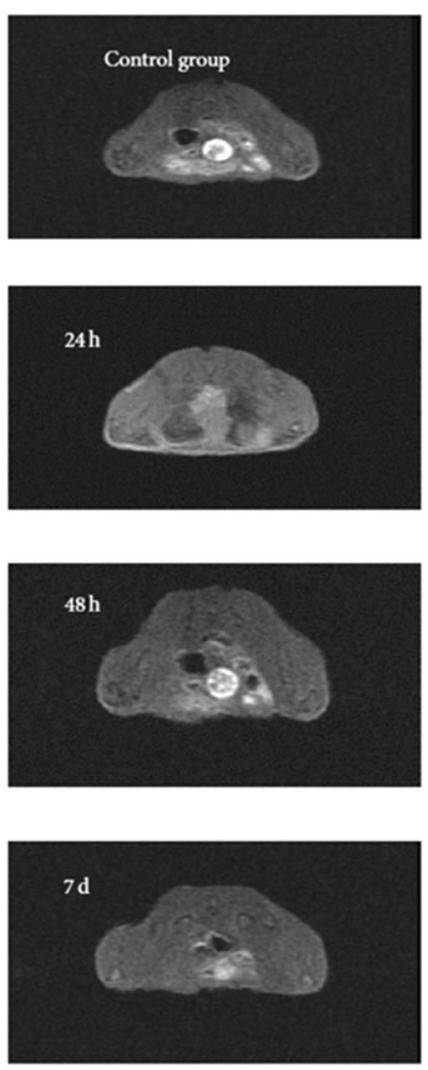

Fig. 4 T2-weighted MR images and in vivo cell tracking. Magnetized cells can be detected as hypointense signals (dots): e is FeNdB magnets $(8 \mathrm{~mm} \times 2 \mathrm{~mm})$ with a magnetic field of $0.5 \mathrm{~T}$. a- $\mathbf{d}$ the SPIONs-MSCs + magnetic field group, MR images were taken at 0 h (a), $24 \mathrm{~h}(\mathbf{b}), 48 \mathrm{~h}(\mathbf{c})$, and $7 \mathrm{~d}(\mathbf{d})$ after cell transplantation, the MRI image in (b) confirms that labeled stem cells (low signal distribution) entered into the trauma center within the first $24 \mathrm{~h}$. e-h SPIONs-MSCs group without magnetic field, MR images were taken at 0 h (e), 24 h (f), 48 h (g), and $7 \mathrm{~d}(\mathbf{h})$ after cell transplantation, the 24-h and 48-h MRI images in this group do not demonstrate a low signal area, corresponding to the skin surrounding the wound

exposure to a magnetic influence, were observed in the skin injury areas of mice 7 days after cell transplantation; however, far fewer positive cells were observed in the SPIONs-MSCs group that did not receive a magnetic implantation. Magnetic implantation, along with injection of SPIONs/GFP-MSCs was found to be safe, as all animals survived and no major signs of tissue injury were observed in vivo by MRI, or ex vivo in the skin tissue.

\section{In vivo optical molecular imaging}

To evaluate the role of the magnetic gradient created by the EMF on the activity of SPIONs/GFP-MSCs, we used in vivo optical molecular imaging as a tracer technique to observe the living cells, which express the fluorescent protein. Fluorescence imaging at 0, 24, and $48 \mathrm{~h}$ demonstrated a trend of targeted SPIONs-MSCs movement under the EMF, which was consistent with MRI results (Fig. 7). However, the optical molecular imaging technique was not as sensitive as MRI.

\section{Discussion}

Our study demonstrated an EMF-targeted approach that promotes the directional movement of SPION-labeled stem cells, enhancing their ability to repair damaged skin tissues. SPION-labeled human MSCs exhibit excellent paramagnetism, and can accurately target lesion locations under the effect of an EMF, thereby increasing stem cell concentrations at the target site. We confirmed that MSCs doubly labeled with SPIONs and GFP reporter gene demonstrate better dry phenotypes in in vivo experiments. We also demonstrated that SPIONs and GFP labeling does not affect MSC proliferation or vitality, as no significant difference in cell viability was observed before and after labeling. The osteogenic and adipogenic differentiation potential is not affected when the amount of SPIONs-labeled MSCs is 90\% [18-21]. In the present study, SPIONs/GFP-labeled MSCs displayed bona fide stem cell features in vitro, similar to that in other studies [22-24]. Weakening of the magnetic flux by exposure to an electromagnetic field, with strength 

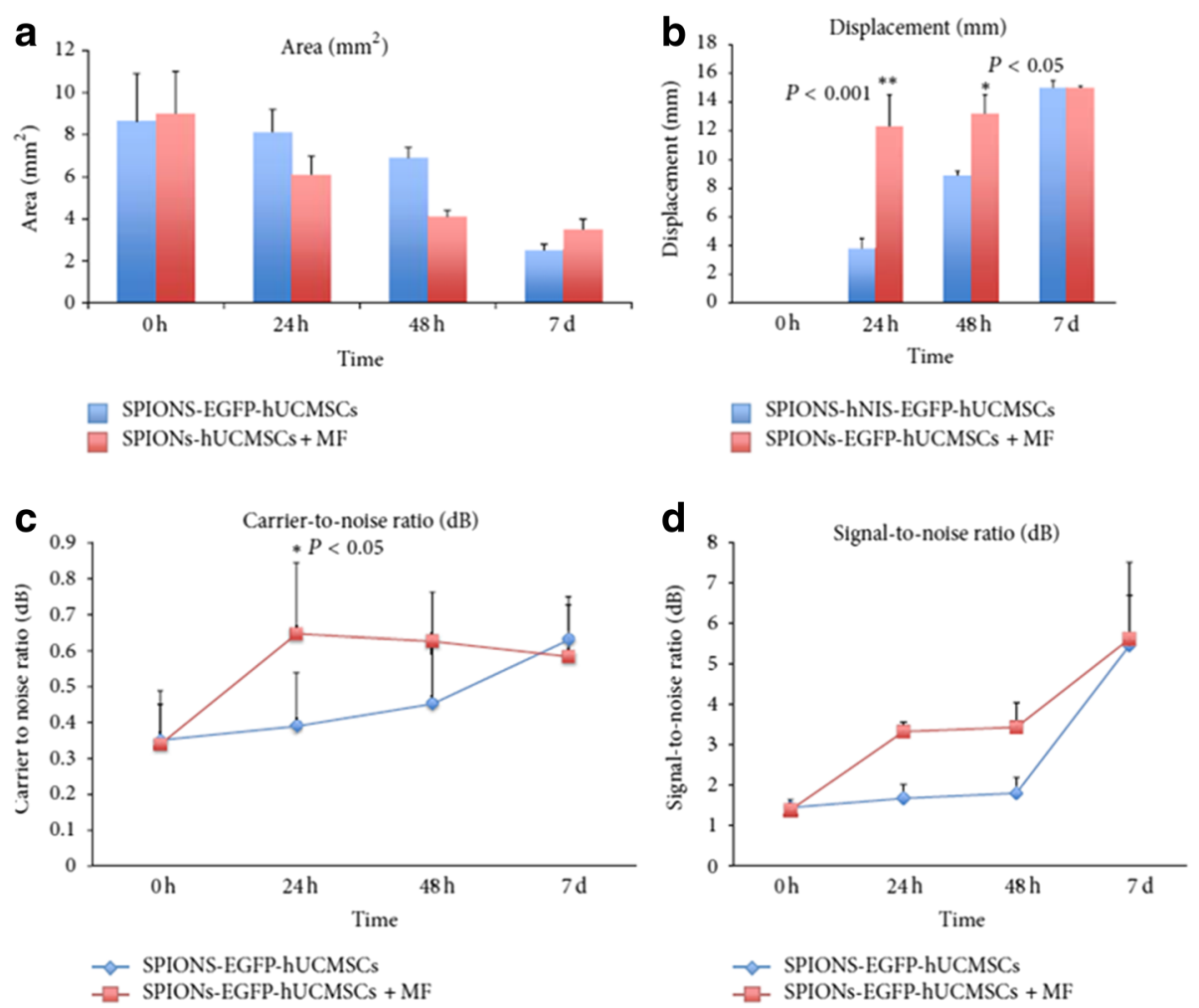

Fig. 5 Parameters of MRI image analysis. a Reduction in the cluster area of labeled MSCs with time. $\mathbf{b}$ 24-h (1 d) and 48-h (2 d) displacement of SPIONsMSCs increased significantly under magnetic field $\left({ }^{*} P<0.05,{ }^{*} P<0.001\right)$. c CNR was the highest at $24 \mathrm{~h}$, and an MRI shows obvious increase in the SPIONs-MSCs + magnetic field group. $\mathbf{d}$ SNR increased in the SPIONs-MSCs + magnetic field group by $24 \mathrm{~h}$, and was high in both groups by $7 \mathrm{~d}$

less than $0.1 \mathrm{mT}$, can promote human umbilical vein endothelial cell proliferation [25]. Exposure of bone marrow-derived MSCs to a $1 \mathrm{mT}$ magnetic field for $1 \mathrm{~h}$ per day has been shown to promote cell proliferation and differentiation at an early stage [26-28]. The characteristics of a permanent magnet are far more complex than that of an electromagnetic field, and the force is distance-dependent; therefore, magnetic field properties were not analyzed in this study. There are very few stem cell studies involving the generation of outside target fields by use of a permanent magnet; however, some studies have successfully applied this method. We put an $8 \mathrm{~mm} \times 2 \mathrm{~mm}$ permanent magnet with a surface residual magnetism of $0.5 \mathrm{~T}$ and a coercive force of $900 \mathrm{KA} / \mathrm{m}$ on the surface of the wound, $1.5 \mathrm{~cm}$ away from the transplanted cells. The magnetic targeting effect was evident from our results. Since the dead labeled-stem cells also release iron-containing nanoparticles, which can be taken up by the surrounding unlabeled stem cells, we utilized MRI and in vivo fluorescence imaging for synergistic monitoring to avoid any false positive results. However, MRI was more sensitive than in vivo fluorescence imaging. MRI demonstrates high spatial resolution; therefore, a small number of cells can be studied and quantified in a very simple manner using this technique. In the present study, in vivo fluorescence imaging was unable to track cells that had been transplanted for $48 \mathrm{~h}$; however, MRI maintained an excellent tracer capacity until the end of the experiment (up to 7 days). In the case of a 6-h magnetic field exposure each day, both tracer techniques demonstrated that labeled stem cells possess a quick and clear central tendency for magnetic fields. MRI was also very sensitive in demonstrating that a considerable number of stem cells entered the epidermal trauma center within $24 \mathrm{~h}$, and revealed that the maximum displacement of cells extending to the center of the magnetic field was $1.5 \mathrm{~cm}$. Furthermore, using MRI, we also determined that the change in maximum displacement after $48 \mathrm{~h}$ was smaller than that in the first $24 \mathrm{~h}$, with a maximum displacement of $0.3 \mathrm{~cm}$. The MRI technique also demonstrated increased cell proliferation in the wound area on day 7 post-cell transplantation. A directed stem cell homing to trauma centers was observed in mice exposed to an EMF; however, other parameters such as displacement, area, and SNR were significantly reduced as compared to in mice that were not exposed to the EMF.

Mice in the EMF group displayed a distinct advantage in the overall wound healing time. The fact that GFP is expressed only in surviving stem cells was used to 


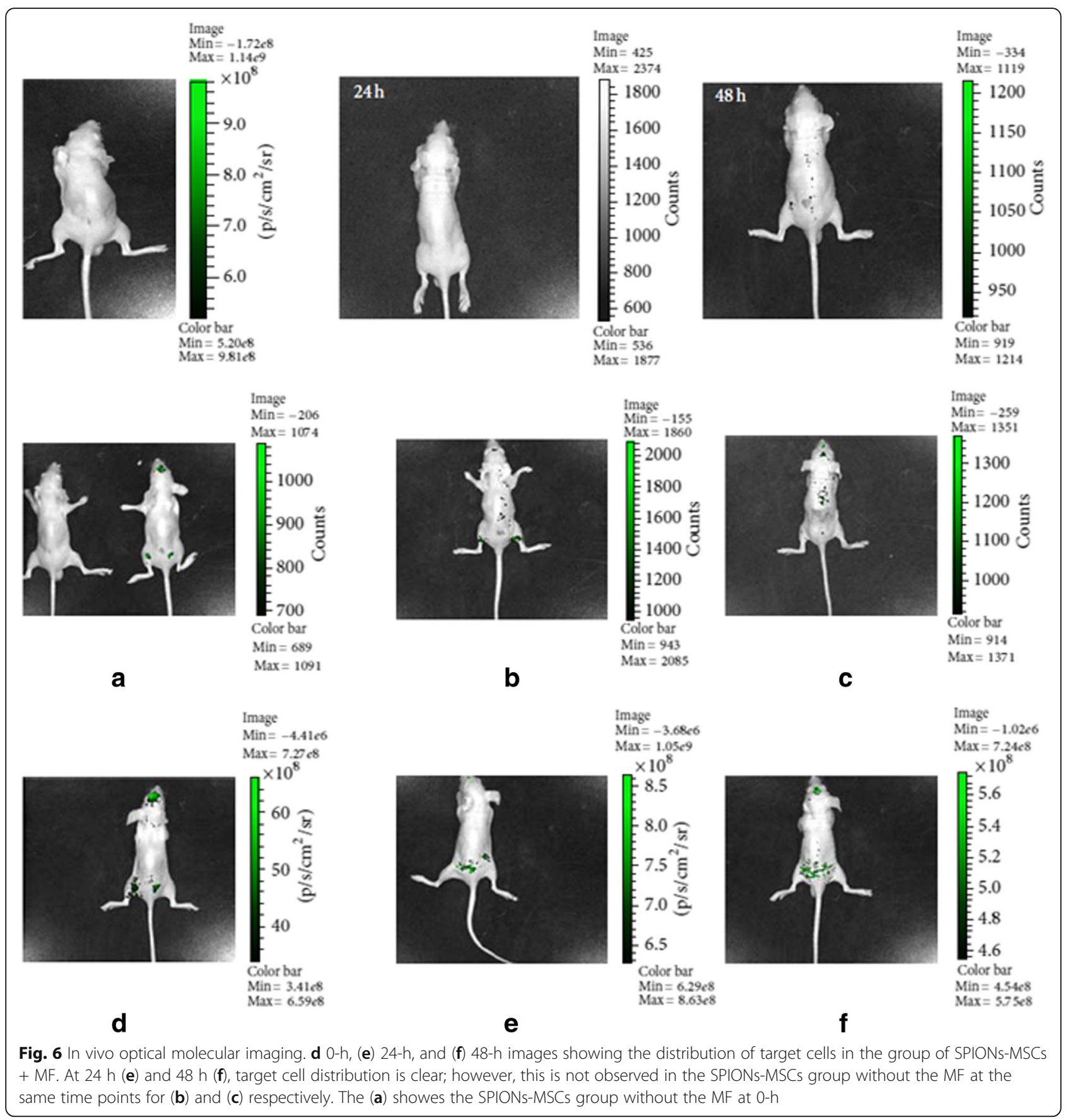

distinguish the false positive cells, and reflect the true state of surviving stem cells. Its advantage was pronounced in pathological tissue sections, and was confirmed by Prussian blue staining. Fluorescence imaging of pathological tissue sections also demonstrated that the aggregation ability of the targeted stem cells under the effect of an EMF was superior to that of control and non-magnetic field stem cell groups.

MRI tracking of SPION-labeled stem cells has so far proven to be an effective technique, resulting in limited concentration-dependent cellular toxicity. A previous study reported a labeling efficiency of $99 \%$, at concentrations of $25-50 \mathrm{mg} \mathrm{Fe} / \mathrm{L}$, without any adverse effects on cell viability, growth, differentiation, and other biological activities [29]. In another study, culture mediums with a SPION concentration of 11.2, 22.4, and $44.8 \mathrm{mg} \mathrm{Fe} / \mathrm{L}$ led to no changes in stem cell viability and proliferation [30]. Proliferation of liver stem cells has been shown to be inhibited by SPIONs at concentrations higher than $100 \mathrm{mg} \mathrm{Fe} / \mathrm{L}$ [31]. It has therefore been recommended 


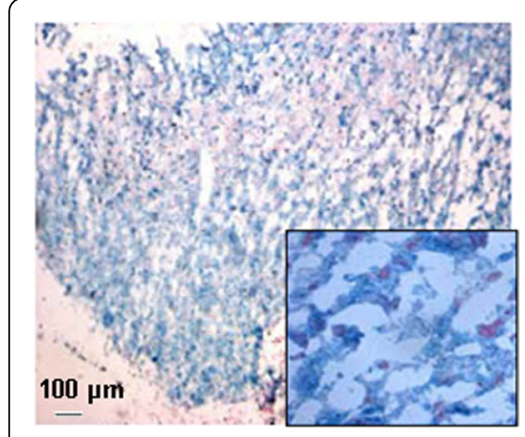

a

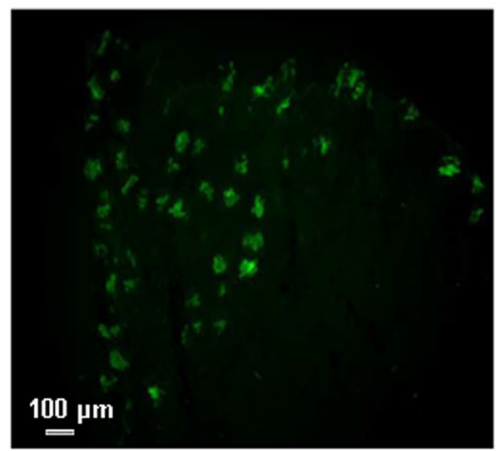

d

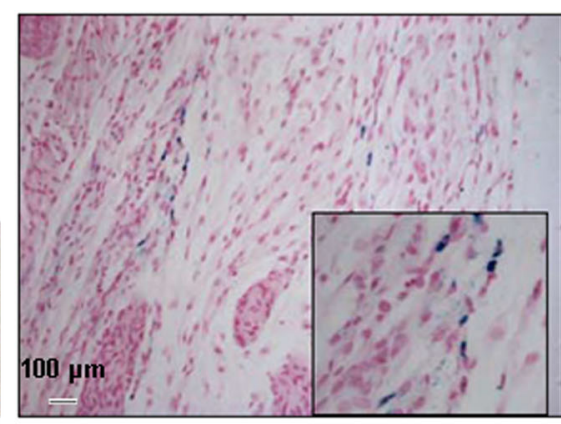

b

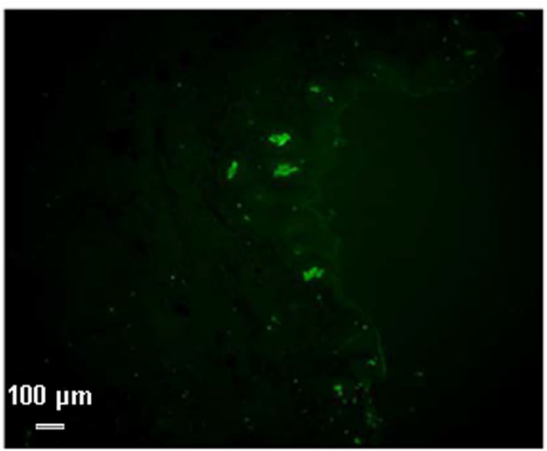

e

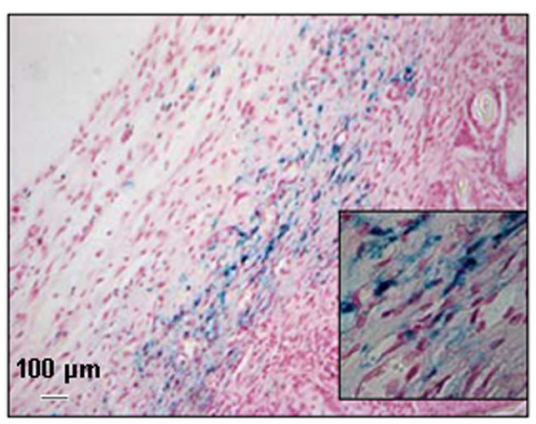

C

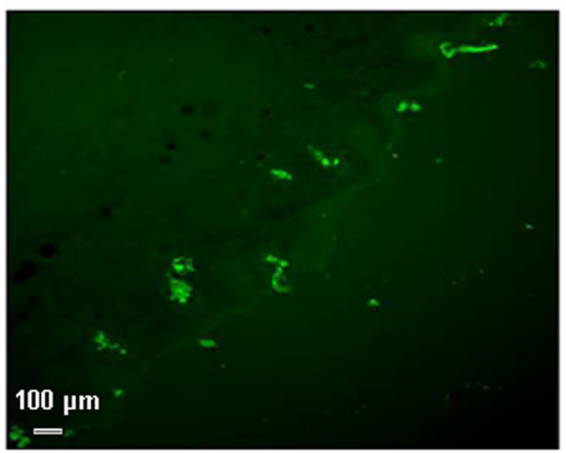

f

Fig. 7 SPIONs and EGFP-labeled human MSCs under magnetic influence identified in skin tissue. c and (f) show only a few positive cells in the group that was not exposed to the magnetic field. $\mathbf{b}$ and (e) show SPIONs and EGFP-labeled human MSCs after $7 \mathrm{~d}$ when the wound healed. $\mathbf{a}$ and (d) show SPIONs and EGFP-labeled human MSCs transplanted subcutaneously in the skin of nude mice at $0 \mathrm{~h}$

that a SPION-labeling concentration of $25 \mathrm{mg} \mathrm{Fe} / \mathrm{L}$ be used [32], which minimally affects the physiological activity of stem cells. In the present study, we used the same SPION-labeling concentration, and observed no changes in cellular morphology or activity.

\section{Limitations}

Our study is limited by the use of relatively few animals, a short duration of the disease, and lack of another control group with only a magnet implant without MSCs injection, to test the effect of magnetic field exposure on skin wound healing. Nevertheless, no histology but the wound area determines whether the skin defect is healed [33]. Moreover, the differentiation potential of SPIONs and GFP-labeled MSCs could have been affected by the iron particles. Further studies are required to explore whether exposure to a magnetic field has the same effect on MSCs from a larger distance and in diseases that are more complex.

\section{Conclusions}

In summary, SPION-labeled stem cells are excellent and safe magnetization and tracer agents, and together with exposure to an EMF generated by permanent magnets, they can be used as a new method of magnetic guidance of targeted stem cells. This method was shown to be safe and effective by MRI and fluorescence analysis of tissue sections. The findings of this study provide a platform for the development of stem cell targeted therapies, and can be further applied for drug and gene targeted therapies.

\section{Additional files}

Additional file 1: Figure S1. The Effect of different external magnetic field exposure time on stem cells was tested by MTT (A), western-blot testing apoptosis marker $(B, C)$. (a) MTT testing showed when the SPION/ GFP positive MSCs exposure $6 \mathrm{~h} / \mathrm{d}$ under magnetic field the cell viability increased, and (b, c) apoptosis marker were low. (TIFF 134 kb)

Additional file 2: Figure S2. Mice were anesthetized with isoflurane (4\% induction, $1.5 \%$ maintenance), and a small permanent neodymium (FeNdB) magnet $(8 \times 2 \mathrm{~mm})$ with a magnetic field of $0.5 \mathrm{~T}$ was put on the wound of mice for $6 \mathrm{~h} /$ day. (TIFF $1394 \mathrm{~kb}$ )

Additional file 3: Figure S3. The distributions of cell growth. (TIFF $589 \mathrm{~kb}$ )

\section{Abbreviations}

bFGF: Basic fibroblast growth factor; CNR: Carrier-to-noise ratio; EMF: External magnetic field; FBS: Fetal bovine serum; GFP: Green fluorescent protein; HUCMSCs: Human umbilical cord-derived MSCs; MRI: Magnetic resonance imaging; MSCs: Mesenchymal stem cells; SD: Standard deviation; SNR: Signalto-noise ratio; SPIONs: Superparamagnetic iron oxide nanoparticles; WJ-MSCs: Wharton's Jelly of the human umbilical cord-derived MSCs 


\section{Acknowledgments}

This study was supported by grants from the Science and Technology Planning Project of Guangdong Province, China (\#2016A040403054). And Important Guangdong Province Science \& Technology Specific Projects (\#2003A3080501).

\section{Availability of data and materials}

The authors confirm that, for approved reasons, some access restrictions apply to the data underlying the findings. Data are available from the Jinan University. Institutional Data Access/Ethics Committee for researchers who meet the criteria for access to confidential data. The restriction on these data showed that the data should be used in nonprofit research, not permit in commercialized research. The contact information for the Jinan University Ethics Committee list as: Address: West 601, Huangpu road, School of medicine, Jinan University Ethics Committee, Jinan University, Guangzhou, 510,632, China. Tel: 86-20-85,224,045.

\section{Authors' contributions}

$\mathrm{HX}$ designed the study and the experiments. YM, CS, BH, JG and XZ performed data collection; XZ, JL, CL and XL analyzed the date. YM and CS wrote the manuscript. HX supervised the writing of the whole paper. All authors read and approved the final manuscript.

\section{Competing interests}

The authors declare that they have no competing interests.

\section{Consent for publication}

Not applicable.

\section{Ethics approval and consent to participate}

All details of this study were approved by the regional ethical review board (Jinan University and Shenzhen people's hospital).

\section{Publisher's Note}

Springer Nature remains neutral with regard to jurisdictional claims in published maps and institutional affiliations.

\section{Author details}

'Department of Nephrology, the First Hospital Affiliated to Jinan University, No. 613 Huangpu West Road, Guangzhou 510630, China. ${ }^{2}$ Department of Radiology, the First Hospital Affiliated to Jinan University, No. 613 Huangpu West Road, Guangzhou 510630, China. ${ }^{3}$ Department of Nuclear Medicine, the First Hospital Affiliated to Jinan University, No. 613 Huangpu West Road, Guangzhou 510630, China. ${ }^{4}$ Shenzhen Engineering Laboratory for Genomics-Assisted Animal Breeding, BGI-Shenzhen, Shenzhen 518083, China.

Received: 25 May 2016 Accepted: 15 May 2017

Published online: 26 May 2017

\section{References}

1. Gao Z, Zhang L, Hu J, Sun Y. Mesenchymal stem cells: a potential targeteddelivery vehicle for anti-cancer drug, loaded nanoparticles. Nanomedicine. 2013:9(2):174-84.

2. Schrepfer S, Deuse T, Reichenspurner H, Fischbein MP, Robbins RC, Pelletier MP. Stem cell transplantation: the lung barrier. Transplant Proc. 2007;39(3): 573-6.

3. Fischer UM, Harting MT, Jimenez F, et al. Pulmonary passage is a major obstacle for intravenous stem cell delivery: the pulmonary first-pass effect. Stem Cells Dev. 2009;18(5):683-92.

4. Li L, Jiang Q, Ding G, et al. Effects of administration route on migration and distribution of neural progenitor cells transplanted into rats with focal cerebral ischemia, an MRI study. J Cereb Blood Flow Metab. 2010;30(3):653-62.

5. Jordan A, Scholz R, Maier-Hauff K, et al. Presentation of a new magnetic field therapy system for the treatment of human solid tumors with magnetic fluid hyperthermia. J Magn Magn Mater. 2001;225(1-2):118-26

6. Jun YW, Lee JH, Cheon J. Chemical design of nanoparticle probes for highperformance magnetic resonance imaging. Angew Chem Int Ed Engl. 2008; 47(28):5122-35.
7. Song HT, Choi JS, Huh YM, et al. Surface modulation of magnetic nanocrystals in the development of highly efficient magnetic resonance probes for intracellular labeling. J Am Chem Soc. 2005;127(28):9992-3.

8. Lewin M, Carlesso N, Tung CH, et al. Tat peptide-derivatized magnetic nanoparticles allow in vivo tracking and recovery of progenitor cells. Nat Biotechnol. 2000;18(4):410-4.

9. Na HB, Song IC, Hyeon T. Inorganic nanoparticles for MRI contrast agents. Adv Mater. 2009;21(21):2133-48.

10. Jordan A, Scholz R, Wust P, Fähling H, Felix R. Magnetic fluid hyperthermia (MFH): cancer treatment with $\mathrm{AC}$ magnetic field induced excitation of biocompatible superparamagnetic nanoparticles. J Magn Magn Mater. 1999; 201(1-3):413-9.

11. Sonvico F, Mornet S, Vasseur S, et al. Folate-conjugated iron oxide nanoparticles for solid tumor targeting as potential specific magnetic hyperthermia mediators: synthesis, hysicochemical characterization, and in vitro experiments. Bioconjug Chem. 2005;16(5):1181-8.

12. Chan DCF, Kirpotin DB, Bunn PA Jr. Synthesis and evaluation of colloidalmagnetic iron oxides for the site-specific radiofrequency-induced hyperthermia of cancer. J Magn Magn Mater. 1993;122(1-3):374-8.

13. Hou CH, Hou SM, Hsueh YS, Lin J, Wu HC, Lin FH. The in vivo performance of biomagnetic hydroxyapatite nanoparticles in cancer hyperthermia therapy. Biomaterials. 2009;30(23-24):3956-60.

14. Manca MF, Zwart I, Beo J, et al. Characterization of mesenchymal stromal cells derived from full-term umbilical cord blood. Cytotherapy. 2008;10(1):54-68.

15. Yin JL, Shackel NA, Zekry A, et al. Real-time reverse transcriptase-polymerase chain reaction (RT-PCR) for measurement of cytokine and growth factor mRNA expression with fluorogenic probes or SYBR green I. Immunol Cell Biol. 2001;79(3):213-21.

16. Kyrtatos $P G$, Lehtolainen $P$, Junemann-Ramirez $M$, et al. Magnetic tagging increases delivery of circulating progenitors in vascular injury. JACC Cardiovasc Interv. 2009;2(8):794-802.

17. Hua Z, Liu L, Shen J, et al. Mesenchymal stem cells reversed morphine tolerance and Opioid-induced Hyperalgesia. Sci Rep. 2016;6:32096.

18. Kostura L, Kraitchman DL, Mackay AM, Pittenger MF, Bulte JW. Feridex labeling of mesenchymal stem cells inhibits chondrogenesis but not adipogenesis or osteogenesis. NMR Biomed. 2004;17(7):513-7.

19. Bulte JW, Kraitchman DL, Mackay AM, Pittenger MF. Chondrogenic differentiation of mesenchymal stem cells is inhibited after magnetic labeling with ferumoxides. Blood. 2004;104(10):3410-2.

20. Soenen SJ, Himmelreich U, Nuytten N, De Cuyper M. Cytotoxic effects of iron oxide nanoparticles and implications for safety in cell labeling. Biomaterials. 2011;32(1):195-205.

21. Balakumaran A, Pawelczyk E, Ren J, et al. Superparamagnetic iron oxide nanoparticles labeling of bone marrow stromal (Mesenchymal) cells does not affect their 'sternness'. PLoS One. 2010;5(7):e11462.

22. Arbab AS, Bashaw LA, Miller BR, et al. Characterization of biophysical and metabolic properties of cells labeled with superparamagnetic iron oxide nanoparticles and transfection agent for cellular MR imaging. Radiology. 2003:229(3):838-46.

23. Chen R, Yu H, Jia ZY, Yao QL, Teng GJ. Efficient nano iron particle-labeling and noninvasive MR imaging of mouse bone marrow-derived endothelial progenitor cells. Int J Nanomedicine. 2011;6:511-9.

24. Yang JX, Tang WL, Wang XX. Superparamagnetic iron oxide nanoparticles may affect endothelial progenitor cell migration ability and adhesion capacity. Cytotherapy. 2010;12(2):251-9.

25. Smith CA, de la Fuente J, Pelaz B, Furlani EP, Mullin M, Berry CC. The effect of static magnetic fields and tat peptides on cellular and nuclear uptake of magnetic nanoparticles. Biomaterials. 2010;31(15):4392-400.

26. Wiskirchen J, Groenewaeller EF, Kehlbach R, et al. Longterm effects of repetitive exposure to a static magnetic field (1.5T) on proliferation of human fetal lung fibroblasts. Magn Reson Med. 1999;41(3):464-8.

27. Nakahara T, Yaguchi $\mathrm{H}$, Yoshida M, Miyakoshi J. Effects of exposure of CHO-K1 cells to a 10-T static magnetic field. Radiology. 2002;224(3):817-22.

28. Wiskirchen J, Grönewäller EF, Heinzelmann $F$, et al. Human fetal lung fibroblasts: in vitro study of repetitive magnetic field exposure at 0.2, 1.0, and 1.5 T. Radiology. 2000;215(3):858-62.

29. Ko IK, Song HT, Cho EJ, Lee ES, Huh YM, Suh JS. In vivo MR imaging of tissue-engineered human mesenchymal stem cells transplanted to mouse: a preliminary study. Ann Biomed Eng. 2007;35(1):101-8.

30. Himes $N$, Min JY, Lee R, et al. In vivo MRI of embryonic stem cells in a mousemodel ofmyocardial infarction. Magn Reson Med. 2004;52(5):1214-9. 
31. Bos C, Delmas $Y$, Desmoulière $A$, et al. In vivo MR imaging of intravascularly injected magnetically labeled mesenchymal stem cells in rat kidney and liver. Radiology. 2004;233(3):781-9.

32. Dai GH, Xiu JG, Zhou ZJ, et al. Effect of superparamagnetic iron oxide labeling on neural stem cell survival and proliferation. Nan Fang Yi Ke Da Xue Xue Bao. 2007:27(1):49-55.

33. Lee H, Hong Y, Kwon SH, Park J, Park J. Anti-aging effects of Piper Cambodianum P. Fourn. Extract on normal human dermal fibroblast cells and a wound-healing model in mice. Clin Interv Aging. 2016;11:1017-26.

Submit your next manuscript to BioMed Central and we will help you at every step:

- We accept pre-submission inquiries

- Our selector tool helps you to find the most relevant journal

- We provide round the clock customer support

- Convenient online submission

- Thorough peer review

- Inclusion in PubMed and all major indexing services

- Maximum visibility for your research

Submit your manuscript at www.biomedcentral.com/submit 